\title{
System Organization of Clinical Symptoms and EEG- Characteristics of Patients with Depressive Disorders in Harmonic Sound Therapy
}

\author{
Elena Grigorieva, Alexey Dyakonov, Valeriy Volovenko \\ Department of Psychiatry, Yaroslavl State Medical University, Yaroslavl, Russia \\ Email address:

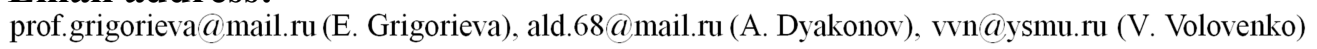

To cite this article:

Elena Grigorieva, Alexey Dyakonov, Valeriy Volovenko. Search of the System Organization of Clinical Symptoms and EEG-Characteristics of Patients with Depressive Disorders in the Course of Harmonic Sound Therapy. Clinical Neurology and Neuroscience.

Vol. 2, No. 3, 2018, pp. 46-55. doi: 10.11648/j.cnn.20180203.11

Received: September 25, 2018; Accepted: December 22, 2018; Published: January 29, 2019

\begin{abstract}
The work is devoted to the search for the systemic organization of clinical symptoms and EEG characteristics of patients with recurrent depressive disorders. It determines the effect of harmonic sound in accordance with the extremes of the maximum and/or minimum EEG spectrum on clinical and EEG characteristics of patients, loosening of stable pathological relationships in order to reduce or eliminate depression. The study involved 58 patients. 35 of them were diagnosed with prolonged and chronic depressive disorder, which to no avail was treated with various antidepressants of the group of SSRIs in combination with benzodiazepines or small doses of atypical (olanzapine, sulpiride) or classical (chlorprotixen, sonapax) antipsychotics. At the time of sound therapy, the drugs were not canceled. 22 patients had repeated depressive episode in the past (two to four weeks ago), untreated. The search for the systemic organization of clinical symptoms and EEG characteristics was carried out using analytical mathematics. In this section factor analysis with factor rotation were used. The positive outcome of the sessions of sound exposure, as well as the reaction to the sound of the last session (no drowsiness or vigor, there is a weakening or elimination of depression), and the number of sessions depended on the removal of the most stable combinations of symptoms (according to the HAM- D scale). They reflected the reciprocity of their own symptoms of depression and anxiety in both groups of patients and made a "hard link" of the system. Other combinations of symptomsflexible links of the system-are less stable. Acting on them in a stable pathological condition (depression) are less radical. Harmonic sound effects cause a significant restructuring of the background EEG observed at the time of exposure and after it in both groups of patients. The features of EEG in patients in complete or incomplete remission, as well as in the absence of the effect of treatment. Neurophysiological "hard link" of the system organization of EEG characteristics could not be identified. An attempt to explain the results is made. Perhaps the "hard link" of the system organization of EEG characteristics is at a different energy level or requires a different mathematical approach.
\end{abstract}

Keywords: Harmonic Sound, Depression, Stable Clinical and EEG Connections

\section{Introduction}

Depressive disorder is a multiple factor process. Understanding of its complexity, variability, ambiguity of prognosis, criteria, and approaches to therapy is increasing. Bioelectric brain activity is also a multiple factor process. It is also characterized by great complexity, variability and nonspecifity. Non-specific is also the reaction to affective stimuli (sound, light, proprioceptive). Many researchers studied EEG noting changes in the amplitude frequency spectrum in healthy people, in people with various pathologies frequency, space time relations in different parts of the brain, etc. A large amount of information, which is in the theoretical vacuum, has been accumulated.

Considering the clinical picture of depression and EEG parameters in depressive patients as a systematic organization of clinical symptoms and EEG characteristics, we assumed that the systems might be similar in some respect. You can detect the principle of isomorphism or other similarity, based on which simulation or modeling is possible. The 
isomorphism sign of the system reflects the 'high link' of the system [2-4], which passes through all stable connections between individual symptoms or EEG characteristics. When affecting the key link it is possible to change the function as a whole. Unstable combinations of symptoms and EEG parameters that change both external and internal influences make a ' flexible link' of the system organization. When influencing it, the function changes, but not always radically and quickly, and not always in the desired direction.

\section{Goal}

The study aimed to identify 'hard' and 'flexible' links in the systemic organization of clinical and EEG depression symptoms, to determine the way the harmonic sound in accordance with the extremes of the maximum and/or minimum spectrum of EEG in patients with the depressive disorder affects his condition and bioelectrical activity of the brain. Changing the mode of sound we can achieve 'loosening' of clinical and neurophysiological stable pathological relationships, contributing to the weakening of the clinical picture of depression.

\section{Method}

Earlier ( 86 healthy people) the general natural reaction of the human brain to the sound harmonic signals of different frequencies was first revealed. The value of frequencies of the harmonic sound influence, at which the reaction of synchronization or desynchronization of brain bio potentials occurs or increases, has been defined by the initial (before the sound effect) EEG of each individual in accordance with the frequencies of extremes of the EEG spectral composition. The synchronization/desynchronization reaction occurs or increases not only under the influence of harmonic sound in accordance with the maximum/ minimum extremes of the baseline EEG, but under the influence of harmonic sound with the frequency multiple of the maximum/minimum extremes with the multiplicity factor of $2 \mathrm{n}$ as well [11-14]. No adverse events were reported. In addition, the sound with the frequency multiple of both the maximum and minimum frequencies has been revealed. It is understandable since there is no absolute polarity in nature. When exposed to this sound the restructuring of the brain bio potentials was more radial. As a consequence of the common factors determined, it has become possible to influence the brain bio potentials in a new way, loosening pathologically stable connections.

57 patients with recurrent depressive disorders (mean number of seizures $3.57 \pm 0.96$ ) aged 18 to 40 years (mean 29 years) underwent the assessment. Of these, 35 were diagnosed with prolonged and chronic depressive disorder, 22- with the depressive episode, repeated, two - four weeks old, untreated. Patients with protracted and chronic depression unsuccessfully took various antidepressants of siozs group along with benzodiazepines or small doses of atypical (mainly olanzapine, sulpiride) or classic (chlorprothixene, sonapaks) antipsychotics. At the time of this study, the drugs were not canceled. Depression in depth in both groups was moderate with the dominant dreary passion without additional symptoms. In prolonged and chronic depression, the affect included a significant apathetic component. The clinical assessment was supplemented with the Hamilton depression assessment scale (17 points).

The study excluded allergic patients, patients with organic brain damage, pregnant, lactating women, and alcohol-drugtoxic addicts.

Background EEG (16 channels according to the international standard), EEG at the time and after sound exposure were recorded in all patients. On their basis the "Brainlog" program carried out a spectral analysis of the EEG, in which there were the extremes of maximum (exceeding the amplitude of the neighboring frequenciesharmonics) and extreme minimum (equal to the amplitude of the neighboring frequencies-harmonics). It is natural that each individual is characterized by its own spectrum of background EEG, its extremes in a given period of time. Consequently, the harmonic sound was individual, had its range of sound effects according to the extremes with a $2^{\text {n }}$ factor. The frequency of harmonic sound was used in the range from 200 to $1500 \mathrm{~Hz}$. The sound was supplied binaurally with the standard volume and duration $(1 \mathrm{~min})$ for all patients. The interval between sounds was 2-3 min. The dependence revealed is typical of all leads, but it was most clearly noted in the occipital and upper-temporal zones, which were used for the analysis. The number of sounds per session was from 4 to 6 . Background EEG, EEG at the time of sound and after it were recorded at each test. Extremes in the background after each sound test changed, as a result the sound supplied changed as well. The Hamilton scale was evaluated before the first sound test and after the session. Each patient, depending on the duration and quality of the therapeutic effect, underwent from 3 to 15 sessions of sound exposure followed by filling in the HAM-D scale as a rule, repeated sessions were the next day after the return of depression.

The search for the systemic organization of clinical symptoms and EEG characteristics was carried out using analytical mathematics. In this paper we used factor analysis with factor rotation. Factor analysis included 2 sections: factor Analysis in the Group of Patients Treated with Psychopharmacological Drugs in Combination with Harmonic Sound; factor Analysis in the Group of Patients Treated with Harmonic Sound Monotherapy.

In both sections, we analyzed:

1) Clinical factors, according to the symptoms of the HAM-D scale, on which therapy effectiveness, the sound response to the last session, and the number of sessions depended.

2) Factors, on which the therapy effectiveness, based on the EEG spectrum (background recording, recording at the time of sound and after the last session) depended.

Comparative analysis of the EEG spectrum in groups depending on the results of the therapy. 


\section{Results}

\subsection{Factor Analysis in the Group of Patients Treated with Psychopharmacological Drugs Along with Harmonic Sound}

The positive result of the sessions, according to the HAMD scale, is associated with the need to influence the "therapeutic niche", reflecting the reciprocity of depressive mood, performance and activity, inhibition combined with anxiety, mental anxiety, critical self-assessment of the individual's condition. This is the first factor covering 31.8\% of the total variance. The second factor - the weight index (16.96\%) indicated that the result of sessions depends on the elimination of middle and early insomnia, the third factor $(9.96 \%)$ indicated hypochondria and weight loss; the fourth one $(7.84 \%)$ somatic anxiety; the fifth $(6,70)$ guilt feelings and late-insomnia. In total, the first five factors covered $73.26 \%$ of the total variance.

The reaction to the sound of the last session (no drowsiness, no increase in vivacity, but just mood improvement) is associated with the same reciprocal relations of symptoms as in the description of the first factor characterizing the positive outcome of the sessions. (The weight index is $31.77 \%$ ). The second factor (the weight ratio of $16.78 \%$ ) indicated that the reaction to the sound is influenced by reciprocal relationships of middle insomnia and genital symptoms. The third factor $(9.87 \%)$ duplicated the third factor of the total sessions and revealed the dependence of the reaction to the sound session on hypochondria and weight loss; the fourth factor $(8.45 \%)$ on General somatic symptoms; the fifth $(6.88 \%)$ on somatic anxiety. The first five factors cover $73.75 \%$ of the total variance.

Effect of the number of sessions on the elimination of depressive symptoms, determined in the first factor $(31,82 \%)$, the same reciprocal relationship as that in the analysis of the positive outcome of the sessions and the type of the reaction to the sound of the last session. The second factor $(16.81 \%)$ recorded: more sessions are required to eliminate the middle insomnia; the third one $(9.22 \%)$ more sessions to eliminate hypochondria and weight loss (the less hypochondria, the less weight loss); the fourth one $(8.42 \%)$ to reduce somatic anxiety; the fifth $(6.37 \%)$ to reduce General somatic symptoms. Overall, the first five factors cover $72.64 \%$ of cases.

Thus, in order to get a positive outcome of the sound therapy combined the drug therapy, to reduce the number of sessions of harmonic sound effects, so that in response to the sound there will be an improvement in mood rather that noticeable increase in vivacity or drowsiness, it is necessary to eliminate the reciprocity of depression and anxiety symptoms. This "hard link" or "therapeutic niche" which you need to affect in the process of therapy is the main. Therapeutic effects broke down some of the links between the symptoms, but there appeared other ones covering a smaller percentage of cases. These are the "flexible links" of the system. They show the effectiveness of treatment, fewer sessions of sound therapy, the reaction to the last session of the harmonic effects that depended not only on the weight stabilization, but also on focusing on their health closely associated with it, which resulted from the description of the third factor in all three analyses. The result of treatment, a smaller number of necessary sessions of harmonic sound were influenced by the relationship of somatic anxiety and General somatic symptoms, which described about $8 \%$ of patients with complete dispersion. The fifth factor with an even smaller weight index characterized the therapy effectiveness that is the elimination of guilt feeling and late insomnia.

\section{Factors Based on the EEG Spectrum}

Rhythm factors of the baseline EEG. The first factor testified $(40.66 \%$ of the total variance) on the relationship between the frequency of delta rhythm of 2.5 and $3.0 \mathrm{~Hz}$; virtually the entire spectrum of the theta rhythm (from 4.5 to $6.5 \mathrm{~Hz}$ ) and low-frequency beta rhythm (of 13.0 to $18.5 \mathrm{~Hz}$ ) and the alpha rhythm with a frequency of $9.0 \mathrm{~Hz}$. The second factor $(12.29 \%$ of the total variance) indicated a close relationship of the alpha rhythm frequency of 10.0 and 11.5 $\mathrm{Hz}$; the third one $(6.51 \%)$ the relationship of the higher beta rhythm frequencies (of 20.5 and $27.0 \mathrm{~Hz}$ ). Other factors had negligible factor loads and were not taken into account in the analysis of the results. In General, three factors characterized the relationship of EEG frequencies in $59.46 \%$ of patients with prolonged and chronic depression with the pharmacotherapy to the harmonic sound. Attention is drawn to the large representation of slow activity in the first factor, characterizing almost half of the patients, weak alpha- and the absence of all waves of high frequency beta range availability in the presence of almost the entire spectrum of low-frequency beta. The second and third factors had low factor loads and were described only by two nearby alpharhythm frequencies (the second factor) and two highfrequencies (the third factor).

\section{Rhythm factors during harmonic sound effects}

The first rhythm factor (the weight index $26.07 \%$ of the total dispersion) testified to the complete elimination of slowwave activity, expansion of the alpha rhythm spectrum (from 10.5 to $12.5 \mathrm{~Hz}$, an increase in the beta rhythm frequency from 18.5 to $21.0 \mathrm{~Hz})$. The second factor $(15.35 \%$ of the total dispersion) was composed only of interrelated frequencies of low-frequency beta rhythm from 14.0 to $16.5 \mathrm{~Hz}$. The third factor $(8.89 \%)$ described the relationship of delta rhythm frequency of $2.0 ; 2.5 ; 3.0 \mathrm{~Hz}$; theta rhythm of $4.5 ; 5.0 ; 5.5$ Hz. The fourth factor, although it characterized only $5.75 \%$ of cases of complete dispersion (the minimum percentage taken into account) revealed the relationship of the interconnected high-frequency rhythms of the beta range. In general, four factors characterized $56.1 \%$ of patients.

Consequently, the harmonic sound effect at the moment of sound causes a significant restructuring of the EEG baseline spectrum in all frequency ranges against the background of pharmacotherapy in patients with prolonged and chronic depressive episodes. The relationship of delta- and thetarhythms with other rhythms determined in the first factor and 
characterizing about half of the cases, "left" for the third independent factor with a small percentage of descriptions.

Factors of EEG rhythms after the harmonic sound effect

The first factor (weight index $24.06 \%$ of the total variance) showed the relationship of only beta rhythm frequencies 19.0-21.0 Hz; the second one (16.29\%) - the relationship of only low frequencies of beta rhythm $(15.0-16.5 \mathrm{~Hz})$; the third one $(10.60 \%)$ - only slow rhythms $(2.5-4.0 \mathrm{~Hz})$. The fourth one selected $(5.75 \%)$ and the fifth factors $(4.89 \%)$, which contained only one EEG spectrum frequency of $8.5 \mathrm{~Hz}$ and $29.0 \mathrm{~Hz}$ respectively. A total of five factors characterized $61.59 \%$ of patients.

Thus, in patients with prolonged and chronic depressive episodes under th psychopharmacotherapy, the first baseline EEG factor, which characterizes about half of the patients, contains a wide range of interrelated frequencies of Delta, theta, beta ranges and to a lesser extent alpha frequencies. These interconnected frequencies of the EEG spectrum are a kind of" therapeutic niche " to be affected, as it is more responsible for the pathological steady state formed at the time of registration. A wide range of interrelated EEG characteristics is most likely to be caused by a disease with a prolonged and chronic course, as well as ineffective treatment with psychotropic drugs. The links of the baseline EEG spectrum frequencies break up under the effect of the sound, and some fragments form separate, but less significant factors, remaining in the place after the sound exposure. This position in the form of the main factor decay containing a greater number of interrelated frequencies of the background EEG spectrum is the loosening of the pathologically stable state, facilitating to the weakening of depression. The main role in loosening is undertaken by slow and fast rhythms. Alpha rhythm links expand their range only at the moment of sound delivery, joining the factor together with the lowfrequency beta rhythm. Further on, their weight index is low, forming an independent fourth factor (only one frequency of $8.5 \mathrm{~Hz}$ is involved), registered after the sound delivery. Perhaps, if in response to the sound, completely new links were formed, rather than fragments of the first most significant factor of baseline EEG, the treatment would be more effective.

\subsection{Factor Analysis in the Group of Patients Treated with Harmonic Sound Monotherapy Effects}

According to the HAM-D scale, the positive effect on the monotherapy with the sound, in the first factor (weight index $-37.42 \%$ ) is associated with the reciprocity (on one pole) of the depressive mood, efficiency and activity, retardation. These symptoms were also noted in the first factor for the patients treated with psychotropic drugs in combination with the sound. Gastrointestinal and genital symptoms are associated with the positive effect as well. On the other pole, there is a kind of mental anxiety and critical assessment of the disease. That is, if there is no psychopharmacological intervention, the range of the sound impact is similar. However, with a somewhat extended range in terms of somatic sphere (gastrointestinal tract and genital symptoms) but with a weakening of reciprocity to anxiety, the impact of the isolated sound on anxiety in terms of the effectiveness of treatment is weaker than in combination with pharmacotherapy. "Therapeutic niche" has somewhat expanded compared to patients of the first group. The second factor $(14.16 \%)$, as in the treatment with the sound along with psychotropic drugs, is associated with sleep disorders. However, if in the first group it concerned early and middle insomnia, in this case it concerns early and late ones: the positive effect depends on the elimination of these interrelated symptoms. The third factor $(10.91 \%)$ pointed to the interconnection of general somatic symptoms and weight loss. the fourth one $(9.32 \%)$ the need to influence guilt feeling; the fifth one $(5.86 \%)$ the need to eliminate the interconnection between the middle insomnia and suicidal thoughts. In total, the first five factors covered $77.67 \%$ of cases of total variance.

The reaction to the final sound session (there is no drowsiness, vivacity, but there is an improvement in mood) is associated (the first factor $-36.88 \%$ ) with the reciprocity of efficiency and activity, depressive mood symptoms, retardation, gastrointestinal and genital symptoms with mental anxiety and agitation. That practically duplicated the first factor of the positive therapy effect in this group of patients with the addition to the mental anxiety or agitation (full compliance with the first factor in patients receiving psychotropic drugs in all factor analyses). In addition, the more severe the symptoms of depressed mood are, the weaker the critical assessment of the individual's condition is. The second factor $(14.17 \%)$, the third $(11.20 \%)$ and the fourth ones $(8.91 \%)$ repeated interrelated combinations of similar symptom factors of the positive outcome of sessions in this group of patients (respectively, early and late insomnia; general somatic symptoms and weight loss; guilt feeling). The fifth factor $(6.11 \% 0)$ is associated with the need to eliminate moderate insomnia with the sound. Five factors describing this patient group cover $77.26 \%$ of total variance.

The number of the sessions administered, in accordance with the first factor $(37.07 \%)$, in the study of only sound effects on patients also depended on the reciprocity of interrelated symptoms of depressed mood, reduced efficiency and activity, gastrointestinal and genital symptoms with interrelated symptoms of mental anxiety and agitation. In the second factor (14.66\%) effects depended on early and late insomnia (duplicated with the second factor of treatment effectiveness and the second factor of reaction to the last session). In the third factor (10.93\%) effects depended on general somatic symptoms and weight loss (factors are duplicated with the factors that depend on the positive effect of the therapy and factors that are associated with the response to the sound therapy only). The fourth factor $(8.63 \%)$ indicated the need for more sessions in the presence of severe somatic anxiety; the fifth $(6.60 \%)$ fewer sessions in the absence of guilt and retardation. Five factors described $77.89 \%$ of patients with complete dispersion.

Thus, in the group of patients treated only with the sound, 
according to the HAM-D scale, with all analyzes, the depression and anxiety reciprocity also occur, but in a slightly different symptoms ratio. For the best effect of the therapy, the response to the sound without subsequent drowsiness and vivacity, more sessions were required to impact not only the reciprocity of the depressive mood, performance and activity, retardation with mental anxiety, but the gastrointestinal tract and genital symptoms as well. The sound was unlikely to be provided. The critical assessment of the individual's condition, anxiety and agitation were included in different groups analyzed and made the "flexible link" of the first factor. An interesting fact is that all three tests are identical to the second (early and late insomnia) and the third (general symptoms and weight loss) factors. The impact of the sound on these symptoms combinations depends not only on the effectiveness of treatment, but the number of sessions, the reaction to them. Therefore, they can also be considered as a "hard link" of the system organization, characteristic of depressive episodes treated only with sound. Other symptoms: somatic anxiety, guilt, suicidal thoughts, medium insomnia were included in different factors with different analyzes, with different weight indexes.

\section{Factors with Account of the EEG Spectrum}

\section{Baseline EEG rhythms factors}

The first factor (a weighting index of $31.65 \%$ of the total variance) shows the relationship of the frequencies only a beta range from 14.0 to $16.0 \mathrm{~Hz}$; from 17.5 to $19.5 \mathrm{~Hz}$; of 20.5 and $21.0 \mathrm{~Hz}$. The second factor $(12,54 \%)$ characterized the relationship of the frequencies of the delta rhythm (1.5 to $2.0 \mathrm{~Hz}$ ) with beta rhythm high-frequency of 23.5 and 24.5 $\mathrm{Hz}$. The third factor (of $8.39 \%$ ) characterized the relationship of the three frequencies of the theta rhythm 4,$5 ; 5,5 ; 7,0 \mathrm{~Hz}$, and the fourth $(6,76 \%)$ delta frequency of 2,$5 ; 3,0 ; 3,5 \mathrm{~Hz}$. The fifth factor $(5,42 \%)$ included one alpha frequency of 11.5 Hz. Overall, five factors described $64.76 \%$ of cases of total variance.

Therefore, in the background EEG in patients without the prolonged and chronic nature of depression, with no prior psychotropic drugs therapy in the first factor with the highest weight index, describing one third of patients, no rhythms but beta ones are involved. In addition, in this group of patients, in contrast to patients with prolonged and chronic depressions in the course of pharmacotherapy, not three, but five main factors (the maximum weight index of 5\%) that form a pathologically stable state were registered.

EEG spectrum factors at the time of the harmonic sound effect

In response to the sound effect, the first factor (weight index $24.58 \%$ of the total dispersion) characterized the relationship of the alpha frequency range $(7.5-8.5 \mathrm{~Hz})$ with the low-frequency beta range of $13.5 \mathrm{~Hz}$ and from 14.5 to 16.5 Hz. The second factor (13.12 per cent) identified the relationship of delta rhythm frequency of 0.5 to $2.5 \mathrm{~Hz}$ with a theta rhythm frequency of $6.0 \mathrm{~Hz}$, beta rhythm high frequency of $26.0 \mathrm{~Hz}$. The third factor $(12,81 \%)$ characterized alpha range frequency of 9,$5 ; 10,0 ; 10,5$; of
$12.5 \mathrm{~Hz}$ with beta of 18,$5 ; 19,5 ; 20,0$; of $20,5 \mathrm{~Hz}$. The fourth factor $(8,47 \%)$ identified the frequency of the theta rhythm from 4.0 to $5.5 \mathrm{~Hz}$ with the beta high-frequency of $25 \mathrm{~Hz}$. The fifth factor $(7,69 \%)$ revealed only interconnected betarhythm high frequency of 27,$0 ; 27,5 ; 29,0 \mathrm{~Hz}$. Overall, all five factors described $66.69 \%$ of patients with complete variance.

So, in the course of harmonic sound there is a significant restructuring of the brain bio potentials, compared with the original, which is reflected in the formation of factors characterizing the relationship of different EEG spectrum frequencies. First, the first factor, describing the fourth and the largest group of patients, narrowed the spectrum of the low-frequency beta rhythm, but there was the alpha rhythm. Other factors were not duplicative either of any factor to the background, or of their rudiments and were of independent nature. The expansion of the relations of the frequencies of the alpha and beta spectra is characteristic.

The EEG factors after the harmonic sound effect

The first factor covered $30.07 \%$ of total dispersion cases and was characterized by the interrelated frequencies of alpha rhythm from 9.5 to $11.5 \mathrm{~Hz}$ and $12.5 \mathrm{~Hz}$ and beta rhythm from 18.5 to $20.5 \mathrm{~Hz}$ and $22.0 \mathrm{~Hz}$. The second factor (the weighting index $19,70 \%$ of the total variance) included almost the whole range of slow-wave activity from 0.5 to 7.0 $\mathrm{Hz}$; the third factor (of 10.86\%) has identified the relationship between the alpha rhythm frequency of 8.0 and $8.5 \mathrm{~Hz}$ with beta high-frequency (gamma) and of 27,5 28,0 $\mathrm{Hz}$. The fourth factor (5.5 percent) joined the different frequencies of the beta range: 17,$0 ; 24,0 ; 29,0 ; 29,5 ; 30,5 \mathrm{~Hz}$. Overall, four factors had a weighting index of $66.13 \%$ of the total variance.

Thus, there is a significant restructuring of the brain bio potentials, determining the weakening of depressive symptoms in patients with depressive episode, without any prior therapy with psychotropic drugs, after harmonic sound effects. A closer connection between the alpha- and betarhythm spectrum of high and low frequencies is registered. The fact of the isolation of a separate second factor determining the connection of the entire spectrum of slow rhythms after sounding, characterizing the fifth part of patients is rather interesting. At the same time, the deltarhythm connections of $1.52 .0 \mathrm{~Hz}$, registered in four factors reflecting the moment of the sound flow are broken.

Factor analysis is based on the identification of interrelated symptom combinations. These dynamic relationships depend on the tasks set, on the types of therapy, on the pathological process dynamics. This is the basis for a systematic approach to the study of any state. Internal links between the symptoms of "hard link" are particularly stable, as it is shown in this paper on the reciprocal relationship of symptoms of depressive mood, reduced performance and activity with mental anxiety, agitation. They always corresponded to the first factors and had the greatest weight index. In addition to the" hard link "of interrelated symptoms, as we show in the work, there are "flexible links" that can not be considered outside the dynamic link with each other and with the 
symptoms of the "hard link".

A "hard link" EEG characteristics accompanying depression, could not be detected. So another attempt to find the identity of EEG parameters in the course of the sound therapy in both groups was made.

\subsection{Comparative Analysis of the EEG Spectrum in the Groups (Background, Moment of the Sound Flow, After Sound) Depending on the Therapy Results (No Effect of the Therapy, Complete or Incomplete Remission)}

Comparative analysis of EEG data carried out prior to the application of sound harmonics (background) of the patients in both groups (mono-, bi-therapy) revealed the following fact. In patients treated with psychotropic drugs the presence of the entire spectrum of frequencies in the Delta range $(0,5-$ 3,$0 ; 3,5 \mathrm{~Hz}$ ) was recorded when compared in complete or incomplete remission cases that occurred later on. In addition, at the exit to the intermission had increased betarhythm frequency of $22.5 \mathrm{~Hz}$. In the group of patients where there was no psychopharmacotherapy, the alpha rhythm of 9.0-10.0 Hz was well represented in cases of intermissions. No other differences between the groups were found. In the case of the absence of the treatment effect in the background sample, on the contrasts, the Delta-range was better represented in the group without pharmacotherapy (frequency of $1.0 ; 2.0$ to $3.0 \mathrm{~Hz}$ ), and with psihofarmakoterapia only at the frequencies of $1.5 \mathrm{~Hz}$ and $3.5 \mathrm{~Hz}$. Moreover, in the group, where further sound monotherapy was carried out, an alpha rhythm with a frequency of $12.5 \mathrm{~Hz}$ was determined in comparison, and in the second group - disparate beta frequencies $(14.5 ; 20.0$; partly $24.5 \mathrm{~Hz}$ ) were determined.

On the background of a harmonic sound exposure in the cases of subsequent remissions in patients with bi-therapy, Delta-activity was preserved almost in full (with the exception of $3.0 \mathrm{~Hz}$ ). In addition, when compared to the monotherapy group, there was a difference in bit therapy in the form of the theta rhythm of 4.0 and $4.5 \mathrm{~Hz}$ strengthening. In the course of monotherapy the frequency of beta rhythm $13.5 \mathrm{~Hz}$ is better represented. In the cases of intermission, there was a significant difference in frequency tuning in response to the sound. Delta-rhythm frequency of $0.5 ; 1.5$ $2.5 ; 3.5 \mathrm{~Hz}$ became characteristic of the patients treated only with the sound and remained in the other group only at frequencies of 0.5 and $3.0 \mathrm{~Hz}$. As in remissions, in the bitherapy group theta rhythm appeared, but the frequency was not 4.0 and $4.5 \mathrm{~Hz}$, but 5.0 and $6.0-7.5 \mathrm{~Hz}$. Theta rhythm frequency of $5.5 \mathrm{~Hz}$ became characteristic of patients with monotherapy. No other frequency differentiating parameters were found in response to the sound. In the cases where there was no effect on the therapy during the sound flow, the difference in Delta - theta - alpha rhythm activity was not revealed. There was a high degree of beta-rhythm in monotherapy at frequencies of 15.0 and $15.5 \mathrm{~Hz}$, and in biotherapy - at a frequency of $29.5 \mathrm{~Hz}$.

After sound exposure of the Delta wave range $(0.5-2.0 \mathrm{~Hz})$ and beta frequency of $22.0 \mathrm{~Hz}$ became more characteristic for patients treated only with the sound in cases of remission. For patients treated with biotherapy distinctive were the frequencies of Delta rhythm of 2.5 and $3.5 \mathrm{~Hz}$, alpha rhythm $-11.5 \mathrm{~Hz}$, and beta rhythm of $24.5 ; 26.0 ; 27.0 ; 32.0 \mathrm{~Hz}$. At the exit from depression to intermission the number of differentiating frequencies in both groups decreases. Of the patients with monotherapy more characteristic are frequencies of Delta rhythm of 1,0-1,5 Hz and beta rhythm of 14.5-15.0 Hz. When harmonic sound and a pharmacotherapy were combined, the frequencies of the Delta range of $0.5 \mathrm{~Hz}$, theta range of 4.5 and $7.5 \mathrm{~Hz}$, and beta range of 25,5 Hz were more significant. In cases of no effect of the therapy given the number of differentiating frequencies is negligible. The Delta-rhythm of $1.5 \mathrm{~Hz}$, beta rhythm of $15,5 \mathrm{~Hz}$; for patients with bi-therapy beta rhythm frequency of $26,5 \mathrm{~Hz}$ is characteristic the frequency characteristic of the patients with monotherapy,

Thus, the nature of the brain biopotential rearrangement depending on the therapy (sound or a combination of psychotropic drugs with the sound), when compared, is different and "affects" mainly the Delta rhythm. So, the presence of the exit to intermission and remission in EEG in concomitant therapy, prior to use of harmonics and almost the entire spectrum of the Delta range can be considered as a positive moment. This is the main differentiating factor when compared to patients treated with the sound monotherapy. This position is preserved both in bi-therapy and in response to a sound, when it comes to remission. After the application of sound harmonics Delta rhythm at the exit to the remission in this group of patients becomes differentiating, in comparison with monotherapy, only at a frequency of $2.5 \mathrm{~Hz}$, while the patients with monotherapy the Delta rhythm is characteristic at a frequency of 1.0 and $1.5 \mathrm{~Hz}$. In the absence of the treatment effect, the frequencies of Delta-range in the course of bi-and monotherapy not are duplicated according to baseline EEG.

Consequently, when exposed to the sound, harmonics (in accordance with the extrema of the maximum and minimum) EEG spectrum with the coefficient $2^{\mathrm{n}}$ of Delta-rhythm, being a baseline sample differentiate between the groups, was characteristic of the EEG in patients with bi-therapy in almost complete frequency range, but at the time of the sound flow and after it the Delta rhythm gradually loses its differentiating position in this group of patients and becomes more characteristic of patients with monotherapy, although in a more narrow frequency spectrum.

Prior the use of harmonics, the theta rhythm was not differentiating among the groups either after intermission or remission, or in the absence of a positive effect of treatment. In the cases of remission during the sound flow it became more common for patients who undergoing the contaminant therapy at the frequencies of 4.0 and $4.5 \mathrm{~Hz}$, and at the frequency of $5.5 \mathrm{~Hz}$ when taking momotherapy. During sounding in cases of intermission theta rhythm in patients with contaminant therapy became differentiating in comparison with monotherapy in a rather wide range of 
frequencies $(5,0 ; 6,0 ; 6,5 ; 7,0 ; 7,5 \mathrm{~Hz})$. A similar pattern persists the after of sounding as well, but on more limited number of frequencies $-4.5-7.5 \mathrm{~Hz}$. In cases of the lack of response to the therapy, the theta rhythm does not act as a differentiating between the groups not only in the background recording, but also during and after the sounding.

Alpha rhythm when comparing both groups as a differentiating one acts weakly. Thus, before the use of sound harmonics in subsequent remissions, alpha rhythm is not differentiating, in intermissions it is better expressed in the group of patients undergoing monotherapy at frequencies of $9.0 ; 9.5 ; 10.0 \mathrm{~Hz}$, and in the absence of the effect of monotherapy at a frequency of $12.5 \mathrm{~Hz}$. During the sound flow alpha rhythm in both groups is not differentiating. After sounding at the frequency of $11.5 \mathrm{~Hz}$ characteristic of the profound undergoing bi-therapy.

High-frequency beta-rhythm of same frequencies is steadily differentiating and characteristic of patients with bitherapyin case of a negative response to treatment both before harmonic influence (background) -20.0 and $24.5 \mathrm{~Hz}$, and during sounding $-29.5 \mathrm{~Hz}$ as well as after sounding $26.5 \mathrm{~Hz}$. In intermissions, it is more vibrant in the background recording at a frequency of $25 \mathrm{~Hz}$ when using a combination of drugs, and ceases to be differentiating during harmonic sounding. After the sound it again becomes separating at a frequency of $25.5 \mathrm{~Hz}$, characterizing patients with bi-therapy. When patients exit after the therapy of depression in remission, the high-frequency rhythms as disconnecting appear only after the sound: in the course of bi-therapy at frequencies of $24.5 ; 26,0 ; 27,0 ; 32,0 \mathrm{~Hz}$ and in monotherapy at a frequency of $22.0 \mathrm{~Hz}$.

Low-frequency beta-rhythm as differentiating between groups acted a little. So, in the absence of the therapy effect it appeared in a narrow range of frequencies in patients with bitherapy in the background sample $(14.5 \mathrm{~Hz})$ in monotherapy - during sounding (15.0 to $15.5 \mathrm{~Hz}$ ), after sounding (to 15.5 $\mathrm{Hz}$ ). In remissions, it is separating in monotherapy at the time of sounding at a frequency of $13.5 \mathrm{~Hz}$; in intermissions-only after harmonic effects at frequencies of 14.5 and $15.0 \mathrm{~Hz}$.

\section{Discussion of Results}

In General, factor analysis in both groups, according to the HAM-D scale, of patients in all analyses (treatment effectiveness, type of response to sound, number of sessions) emphasized the need to reduce the reciprocity of the depressive and anxiety symptoms that corresponded to the dreary and disturbing affects. The existing differences of anxious and dreary affects were pointed out by many authors [12-14]. There are many transitional variants between these States, which confirms the complexity of the provision on "fluidity" of syndromic boundaries in psychiatry [15-17].

The result of reducing the amount of the EEG spectrum frequencies (when comparing groups) in the case of the absence of effect from treatment during harmonic sound and after it (the smaller the reaction to the sound from this part of the patients?) is interesting.
Based on the idea that the Delta rhythm plays a part in the distant aggregation of brain structures, groups fast neuronal activity to consolidate the data received $[1,9,19,21]$, which is essential for the elimination of the depression, it can be assumed that the differentiating Delta rhythm plays a positive role. In bi-therapy, this aggrigation and grouping of neuronal activity is provided by taking psychotropic drugs. In the group of monotherapy this positive aspect when comparing EEG prior to the impact of the sound harmonics is missing. However, in order to ensure the aggrigation of the brain structures, grouping of the neuronal activity to consolidate the information obtained, it is likely to be necessary. Therefore, in the process of sound treatment, there appear some Delta-rhythm frequencies as differentiating in the group where there was no psychotropic drugs dosing.

The idea that the frequencies of delta-range waves have a positive impact on the genesis of complete or incomplete remission in both groups is confirmed by the data in both groups of there is no effect of the therapy. In the background recording, the Delta rhythm in both groups of patients was not dominant, but was broken down as differentiating into separate frequencies; in response to the sound it ceases to be differentiating at all; after sounding only one frequency acts as differentiating, characterizing patients having monotherapy experience.

In response to the sound in cases of prolonged and chronic depression in the course of bi-therapy, with a positive response to the treatment (especially when entering the intermission) there appeared a theta rhythm as a differentiator, when compared with the sound monotherapy. It seems probable that this indicates the fact that this group of patients reacts to the sound with the need for greater operating voltage, more significant grouping of neuronal connections between different areas of the cerebral cortex, hippocampus and frontal cortex $[8,16,17]$ than the group of patients without prolonged and chronic depressive disorders who underwent the monotherapy. After the sound effect, the theta rhythm in a limited amount of frequencies remains differentiating only in intermissions, which provides a more complete exit from depression in patients undergoing concominant therapy. In addition, in groups of patients, a kind of reciprocity of alpha and theta rhythms is found while analyzing comparatively EEG in the background recording, and in response to the sound, and after sounding. They never act as differentiators in complete and incomplete remissions together (or alpha- and theta - rhythm). In cases of negative response to the therapy, both rhythms were not registered as differentiating. The data on the reciprocity of alpha and theta rhythms are consistent with the results of W. Klimesch study [18], who noted the reciprocity in the reaction of alpha and theta rhythms in response to external stimuli (light, sound).

Factor analysis revealed a very interesting situation in the group of patients, where the harmonic sound was given against the background of psychopharmacotherapy. In the background recording, connections of almost entire spectrum of EEG are recorded, which disintegrate (at the time of sounding and after it), at a positive response to treatment 
under the influence of sound, forming new factors with lower weight indexes, including a separate factor combining the slow range of rhythms. When therapy is only a sound that process was not observed. The factors did not duplicate the fragments of the background, but were of independent nature, emphasizing the increasing relationship of alpha and betarange frequencies, the relationship of the beta frequencies of high and low frequencies with each other. On a good response to treatment, an independent factor of interrelated frequencies of slow rhythms of the entire spectrum describing a one-fifth of patients appeared. It is consistent with the data of comparative analysis, where a positive response in monotherapy is accompanied by an increase in slow wave activity.

Overall, the brain in action is a kind of electrochemical apparatus and a harmonic sound influence causes changes in its bioelectrical activity. Changes in the ranges of all EEG frequency spectra recorded (compared to the background) on the sound background and after the sound are defined, and it is difficult to explain all the results got. The assumption that at a large accumulation of factors it is possible to state theoretical vacuum is confirmed.

Apparently, at the time of the mathematical analysis the following contradiction appears: on the one hand, we obtained a negative response to monotherapy with harmonic sound in some patients, if there is a slow activity (its diffuse increase in depression was found $[15,27,31]$; on the other hand, a positive response obtained is there, is an independent factor consisting of slow wave activity. Most likely, this is due to the fact that we are talking about interconnected, rather than unrelated characteristics of Delta waves, which had to be broken ("therapeutic niche") to get a positive answer. Relying on the idea (as indicated) that Delta activity plays a role in the distant association of brain structures, groups neuronal activity quickly in order to consolidate the data obtained, and theta participates in the functional association of subsystems connected with the formation and reproduction of mnestic images, controls the hippocampal area and the frontal cortex in the formation of long-term memory, it can be assumed that it is the interaction of interconnected slow rhythms that provides the desired result. It is the merger of rhythms into more or less permanent systems that ensures the rupture of pathological connections formed in the course of the development of a depressive disorder. When comparing the EEG spectrum between groups, depending on the results of the therapy, a special positive role was revealed in the formation of the intermission or remission of the Delta rhythm. However, it should be emphasized that a clear "hard link" of the systemic organization of brain biopotentials affecting the result of the therapy (as revealed in the course of clinical data analysis) was not obtained.

In the study, we used EEG spectra with a frequency of 0.5 $\mathrm{Hz}$. As a result, with all the mathematical approaches applied, intermediate rhythms 3,$5 ; 7,5 ; 12,5 ; 20,5 ; 25,5$ etc., were determined. This emphasizes the assumption of a smooth transition of one frequency to another and the convention of the allocation of the existing frequency range. The convention of the allocated frequencies is confirmed with the data of the slow wave complication and, perhaps, alpha waves with gamma-rhythm layered on them (there is an expansion of the gamma-rhythm spectrum up to $200 \mathrm{~Hz}$ and even up to $1000 \mathrm{~Hz}$ [8], and, on the contrary, all ranges from the Delta-rhythm frequency and above are layered on slower waves up to $0.01 \mathrm{~Hz}$ [29].

None of the researchers has managed to determine a neurophysiological "hard link" so far. This can be caused by different reasons. There is a catch phrase "the Brain always opposes the brain". Well-known is the duality of the brain, the duality of its richest mechanisms, the two faces of Janus. First of all, these are protection mechanisms as a reasonable ban on the disease. It is still believed that any depression is a kind of the defense, but it is hard to say when the protection ends and the disease begins. To connect all factors at a multiple factor disorder, including genetic, neurochemical, environmental and others, to allocate from them a system of "rigid links", to connect these bricks together is the most difficult task. In addition, we lack the knowledge about psychic energy. It is clear that the work of the brain is not limited to electromagnetic oscillations, which are actively studied (super-slow potentials, EEG spectrum, gammarhythms). Psychic energy is called in different ways and is associated with the masses of particles many times less than the mass of the electron (quantum energy, quasi-potential, neutrino). The other candidates are still in theory - axion, wimp. For comparison, neutrino, the elementary particle selected, has a mass of 1000 times less than the electron. Perhaps the neurophysiological "hard link" is focused on the other energy level that is not currently available for our study. Other mathematical approaches are likely to be required.

A systematic approach to the study of depression, as well as the desire to interrupt the pathologically stable state with the harmonic sound effects applied in this paper, is an attempt to lay another brick in understanding of clinical and clinical-physiological mechanisms of depressive disorders, being well aware of their multiple factor nature.

\section{Conclusion}

1. It was found that the positive result of therapy (according to the NAM-D scale) both in patients treated with psychotropic drugs and sound, and in patients treated only with sound, as well as the reaction to the sound of the last session of sound effects (drowsiness, cheerfulness or simply improvement of mood), a smaller number of sessions depended on the successful elimination of steadily interrelated clinical symptoms ("hard" link of the system organization), reflecting the reciprocity of the actual depressive symptoms with anxiety.

2. Less stable combinations of systemic organization of clinical symptoms ("flexible" links) are not duplicated in both groups, neither with a positive response to therapy, nor with different reactions to the sound of the 
last session, nor with the number of sessions. Breaking up, they do not lead to a radical elimination of depression, as new interrelated combinations of clinical symptoms are formed, but with a smaller percentage of cases of complete dispersion.

3. Stable connections ("hard" link) of the system organization of EEG-characteristics are not revealed. In both groups of patients analyzed, harmonic sounding causes a significant restructuring of the EEG spectrum both at the moment of sounding and after it. However, the relationship of EEG characteristics is extremely unstable, variable; expressed variability was observed in responses to therapy.

4. In cases of no effect of treatment in both groups, the comparative analysis significantly reduced the number of differentiating frequencies during and after harmonic sound, which which corresponds to less response to sound.

5. A positive role in the formation of complete or incomplete remission of Delta-band waves was revealed. In General, the restructuring concerns all analyzed EEG rhythms, but it was not possible to catch any system at this stage of the study using factor analysis with the rotation of the factor. The situation is confirmed: with a large number of facts, a theoretical vacuum is established.

\section{References}

[1] Bekhtereva N. P. "Delusions of the brain and labyrinths of life".-S. Pb.-1999.

[2] Bekhtereva N. P. "Human brain-biocomputer" / / Doc. World Wide. Congress. "The outcome of the Millennium" in 2008.p. 1-9. file // E. EEG-sound $\backslash$ mysteries of the brain.

[3] Bekhtereva N. P. "Neurophysiological aspects of mental activity".-L.:Nauka. 1971.- 119s.

[4] Grigorieva E. A., A. A. Pevzner, Shakhnazarov S. S., "Automating the detection of synchronization and desynchronization of the EEG" // South Siberia. scientific. bulletin. Science.- techne. Gah. Section I. Math. modeling and automation. in the industry. and scientific. research. -2012. - p. 25-28.

[5] Grigorieva E. A., Pevzner A. A., Shakhnazarov S. S. "System of synthesis of acoustic influence for correction of psychophysiological state". SB. work. Yaroslavl innovation and technology forum "Eureka". The best innovators. Projects. -2012. - p. 22-23.

[6] Grigorieva E. A., Pevzner, A. A., Dyakonov, A. L., "Dynamics of a depressive disorder in response to the harmony sound in accordance with the extrema of the maximum and minimum of the spectrum of the background EEG" // J. of social. and wedge. psychiatrist.-2013. -3.- p. 60-67.

[7] Grigorieva E. A., Pevzner, A. A., "Method of exposure on the body”. Pat. RF №2143939. Bul. INF. No. 1. 10.01.2000.

[8] Pevzner A. A., Grigoriev E. A., Dyakonov, A. L., Pevzner, L. A., Us Pat. RF \# 22965995, A61N7/00, A61M21/00, A61B5/94. "The method of influence on the body". Bul. No.

\section{9.-10.04.2007.}

[9] Pevzner A. A., Grigorieva E. A., Shakhnazarov S. S., Dyakonov A. L., Cornavin A. V. "The Method of treatment of mental disorders" // Patent for the invention №2462181. 21.03.2011.

[10] Pevzner A. A., Grigorieva E. A., Shakhnazarov S. S., Dyakonov A. L., Cornavin A. V. "The Method of determining synchronization and desynchronization of bioelectric activity of the brain”. The patent for the invention №2454172. - 03.02.2011.

[11] Pevzner A. A., Grigorieva E. A., Dyakonov A. L., "Bundesrepublic Deutschland". Urkundeaber die Eintragung des Gebrauchsmusters № 202013002 906.4.-2013.

[12] Olesen I., Gustavisson A., Svensson M., Wittchen H.-U., Jonsson B. "On behalf of the CDBE 2010 study group and the European Brain Council. The economic cost of brain disorders in Europe" // Eur. J. Neurol. -2012. -19.-p. 155-162.

[13] Somers J. M., Goldner E. M., Waraich P., Hsu L. "Prevalence and incidence studies of anxiety disorders: a systematic review of the eiterature" // Can. J. Psychiatry.-2006.-51.-p. $100-113$.

[14] Vetrogradova O. P. "General principles of treatment and prognosis of depression" // Proc. scientific. works "Psychopathological and pathogenetic aspects of prognosis and therapy of depression".-M. -1985. - p. 5-10.

[15] Bolton J. M., Pagura J., Enns M. W., Craut B., Sareen J. A. "Population-based longitudinal study of risk factors for suicide attempts in major depressive disorder" // J. Psychiatr. Res.-2010.-44.-13.-p. 817-826.

[16] Dementieva N. F. "Psychopathology of anxiety" // J. neuro. psychiatrist. -1980 . -12 . S. 1856-1861.

[17] Lehmann H. E. "The clinican's view of anxiety and depression" // J. Clin. Psychiatr. -1983.-44.-8.-p. 3-7.

[18] Anokhin P. K. "Biology and neurophysiology of the conditioned reflex". -M.: Science.-1968.- 547s.

[19] George M., Schlaefer T. "Brain stimulation treatments for depression" // Word J. Biolig. Psychiatry. 2014.-15.-p.167168.

[20] Korobeinikova, I. I. "The Relationship of the spatial synchronization of potentials in the theta range of the EEG of a person with different success of visual-spatial problems" // J. physiology. chelov.: -2011. -37.-5.- p. 26-34.

[21] Melnikova T. S., Sukarsi E. E., Kovalev A. V., Mosolov S. N. "The dynamics of spectral characteristics of EEG during the application of transcranial magnetic stimulation in patients with resistant depression" // J. neurology. psychiatrist.-2015. 8. - p. 35-41.

[22] Edelman G. M., "The remembered present. A biological theory of conciouness". New York: Basic Books Inc.-1993.

[23] Grigoriev Yu. G. "Man in the electromagnetic field (the essence of the situation, the expectation of bioeffect and hazard assessment)" // Radiation biology. Radioecology. -1997. -37. -4.S. 690-702.

[24] Iluchenok I. R. "The Difference of the frequency characteristics of the EEG in the perception of positive emotional and neutral words" // Zh. Vyssh. nervous. deyat.1998. -48. - p. 447 -450. 
[25] Ivanitsky. A. M. "The Recognition of the type solved in the mind of the task of a few seconds the EEG using learner classifier" // Zh Vyssh. nervous. deyat. -1997.-4.- p. 743-747.

[26] Klimesch W. "EEG alpha and theta oscillation reflect cognitive and memory performance: a review and analysis" // Brain Res.-1999.-29.-2-3.-p. 169-195.

[27] Grin-Yatsenko V. A., Baas, Ponomarev V. A., Kropotov Yu. D. "Electroencephalograms in patients with initial manifestations of depression. Independent component analysis" / / Fiziol. chelov. -2011.-31.-1.- p. 45-55.
[28] Roemer R. A., Shagass C., Dubin W. R. et al. "Quantitative EEG in elderly depressive" // Brain. Topog.-1992.-p. 285-291.

[29] Volf N. V., Passynkova N. R. "EEG mapping in seasonal affective disorders" // J. Affect. Disorrd. -2002.-72.-p. 61-69.

[30] Edelman G. M., Gaily J. A. "Nitric oxide: Linking space and time in the brain" // Proc. Natl. Acad. Sci. USA. -1992. -89.24.-p. 11651-11652.

[31] Steriade M., "Grouping of brain rhytms in corticothalamic systems" // Neuroscience.-2006.-137.-4.-p. 1087-1106. 